\title{
First Structural Characterization of the Chloroplast Division Accessory Protein ARC6
}

Min Woo Sung ${ }^{1}$, Stanislav Vitha ${ }^{2}$ and Andreas Holzenburg ${ }^{1,2,3}$

${ }^{1}$ Department of Biology, Texas A\&M University, College Station, TX 77843-3258, USA

${ }^{2}$ Microscopy \& Imaging Center, Texas A\&M University, College Station, TX 77843-2257, USA

${ }^{3}$ Dept. Biochem. \& Biophysics, Texas A\&M University, College Station, TX 77843-2128, USA

Chloroplasts and all plastids have to divide in order to maintain their numbers in proliferating cells. The integral membrane ARC6 is one of the key regulatory proteins for division of plastids in Arabidopsis thaliana $[1,2]$. In the absence of ARC6, chloroplast division is completely blocked and cells contain only 1-2 grossly enlarged chloroplasts. The N-terminal portion of ARC6 is in the plastid stroma, where it interacts with the C-terminus of the plastid division protein FtsZ2 and serves to stabilize and anchor FtsZ1-FtsZ2 assemblies [1,3]. Although a large body of data is available on FtsZ ring formation and chloroplast division $[4,5]$ no structural data have been reported for ARC6 to date.

Here single particle analysis is presented as a first step towards its structural characterization. Pichia X33 strain expressing the truncated form of mature ARC6 (D54-D450 - stromal portion at the Nterminus) was used in this study. Freshly purified protein was subjected to gel permeation chromatography yielding a major and a minor peak at $16.8 \mathrm{ml}$ and $18.0 \mathrm{ml}$, respectively. A comparison with calibration standards suggested that the major peak corresponds to $\sim 100 \mathrm{kDa}$ and the minor peak to $\sim 40 \mathrm{kDa}$. In addition, the major peak was also accompanied by a shoulder towards larger molecular masses. A major peak fraction containing $70 \mu \mathrm{g}$ protein $/ \mathrm{ml}$, was used for specimen preparation. Protein was adsorbed (10s) onto carbon-coated grid and negatively stained with a $2 \%(\mathrm{w} / \mathrm{v})$ aqueous solution of uranyl acetate following essentially the method described [6]. Specimens were observed in a JEOL 1200 EX TEM operated at an acceleration voltage of $100 \mathrm{kV}$. Calibrated electron micrographs were recorded using a 3k slow-scan CCD camera (model 15C, SIA). Single particle averaging was carried out using routines in the EMAN software package [7] as well as ImageJ (http://imagej.nih.gov/ij).

Overall the sample revealed a monodisperse behavior without significant trends towards aggregation (Fig. 1). Different projections were observed, many of them essentially spherical in shape without immediately distinct features. However, one readily discernible projection observed can be described as two parallel densities as highlighted in Fig. 1a-c. Measurements on these projections suggest that the parallel stack of densities represents a dimeric form of ARC6. Assuming each of the two parallel densities takes the shape of a prolate ellipsoid with half-axis dimensions $a=5 \mathrm{~nm}$ and $\mathrm{b}=1.6 \mathrm{~nm}$ gives the monomer a volume of $53.6 \mathrm{~nm}^{3}$ and a molecular mass of $44.2 \mathrm{kDa}$ as per [8]. The latter is in excellent agreement with the calculated molecular mass for a monomer $(43.8 \mathrm{kDa})$. Another class of distinct projections are shown in Fig.1a,d,e. This projection is triangular in shape and the dimensions agree with an arrangement comprised of three dovetailed protomers forming an equilateral triangle, i.e. constituting a trimeric form of ARC6. Further analysis and 3D reconstruction of ARC6 are challenged by the simultaneous presence of different oligomeric states in the sample, and this will be addressed by more finely dissecting the fractions obtained by gel permeation chromatography. Full-length ARC6 may also alleviate the presence of different oligomeric states and thus permit a more straightforward approach for single particle analysis. In addition, it is conceivable that ARC6 is better behaved in the presence of FtsZ2. Data for the different approaches are presented and outcomes discussed. 


\section{References}

[1] Glynn, J. M., Froehlich, J. E., and Osteryoung, K. W. Plant Cell, (2008) 2460.

[2] Vitha, S., Froehlich, J. E., Koksharova, O., Pyke, K. A., van Erp, H., and Osteryoung, K. W. Plant Cell, (2003) 1918.

[3] Maple, J., and Moller, S. G. Ann Bot, (2007) 565.

[4] Adams, D. W., and Errington, J. Nat. Rev. Micro., (2009) 642.

[5] Okazaki, K., Kabeya, Y., and Miyagishima, S. Y. Plant Signal Behav, (2010) 164.

[6] Valentine, R. C., Shapiro, B. M., and Stadtman, E. R. Biochemistry, (1968) 2143.

[7] Ludtke, S. J., Baldwin, P. R., and Chiu, W. J Struct Biol, (1999) 82.

[8] Holzenburg, A., Jones, P. C., Franklin, T., Pali, T., Heimburg, T., Marsh, D., Findlay, J. B., and Finbow, M. E. Eur J Biochem, (1993) 21.

[9] The support of the Division of Research (Texas A\&M University) is greatly acknowledged.

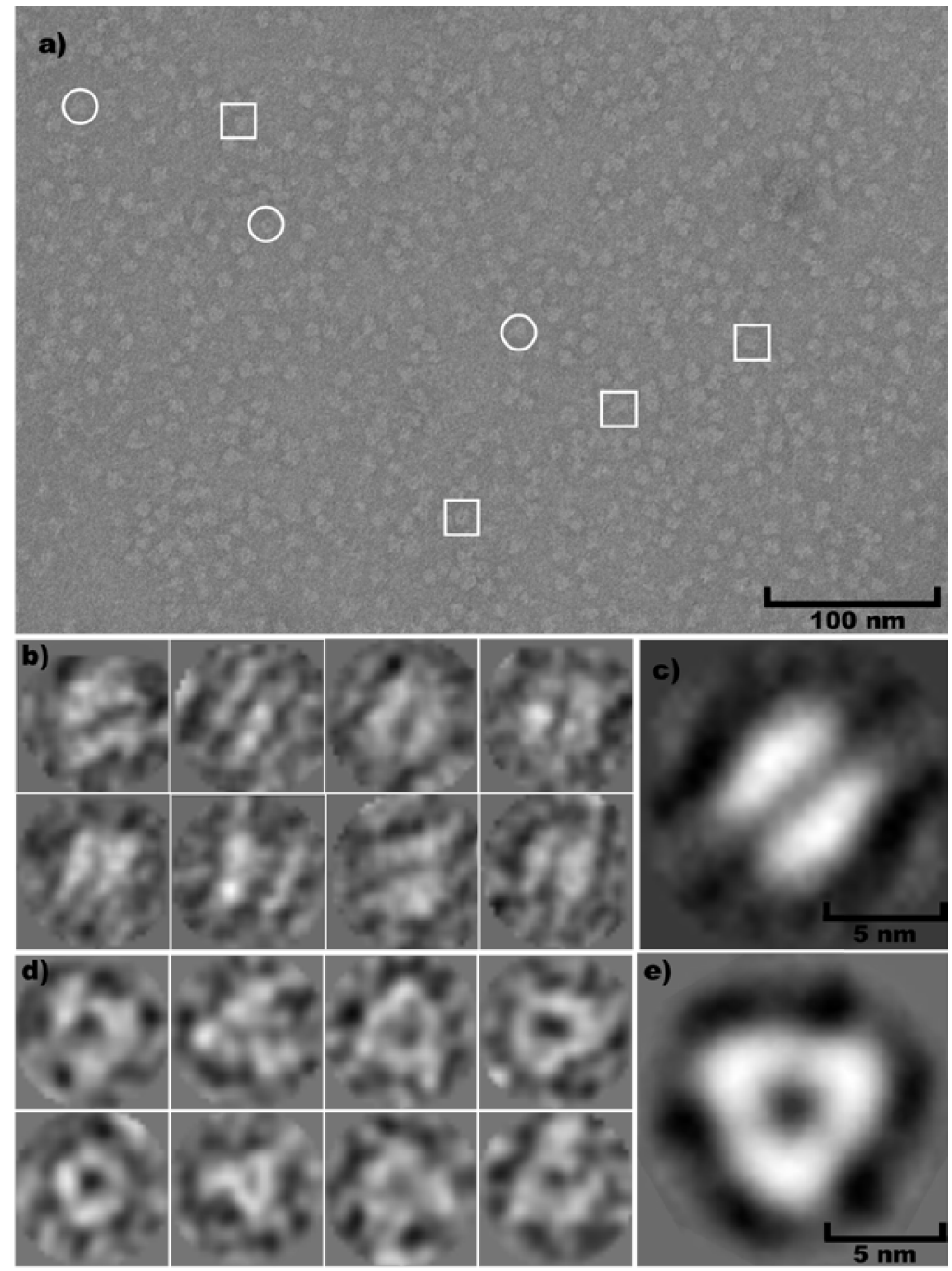

Figure 1. TEM analysis of negatively stained ARC6 molecules. (a) Overview electron micrograph with projections corresponding to dimers and trimers highlighted by boxes and circles, respectively. (b) Gallery of raw dimers and corresponding averaged projection ((c); additionally symmetrized). (d) Gallery of raw trimers and corresponding, symmetry-enhanced average (e). 